\title{
Circular-polarization independence of microwave-induced resistance oscillations and the zero-resistance state
}

\author{
Shenshen Wang and Tai-Kai Ng \\ Department of Physics, Hong Kong University of Science and Technology, Clear Water Bay Road, Kowloon, Hong Kong
}

(Received 9 September 2007; revised manuscript received 19 January 2008; published 17 April 2008)

\begin{abstract}
The immunity of microwave-induced magnetoresistance oscillations and corresponding zero resistance regions to the direction of (circular) polarization of microwaves is studied in this paper. We propose that a spontaneous circular motion of the whole electron fluid would stabilize the system and minimize the polarization sensitivity of the oscillatory dc resistance. Results of a self-consistent calculation capture the qualitative features of the experimental observation.
\end{abstract}

DOI: 10.1103/PhysRevB.77.165324

PACS number(s): 73.40.-c, 73.50.Fq, 76.40.+b, 78.67. $-\mathrm{n}$

\section{INTRODUCTION}

The observation of the "zero-(dc)-resistance state" (ZRS) in high mobility two-dimensional electron systems (2DES) under magnetic field and microwave (MW) radiation presented a surprise to the physics community. ${ }^{1,2}$ When the system, under crossed uniform magnetic field and small dc bias, is irradiated by microwaves of sufficient intensity, the longitudinal (dissipative) resistance $R_{x x}$ develops a strong oscillatory dependence on the magnetic field. At low temperature and high radiation power the minima of the oscillations evolve into the ZRS.

Intense interest in the physics community has been aroused by this unusual nonequilibrium phenomenon. To identify the microscopic mechanism for the MW-induced resistance oscillations (MIRO) and ZRS, previous researchers proposed the picture of photon-excited transport assisted by short-range scatterings ${ }^{3-5}$ and later the scenario of MWinduced oscillations in the nonequilibrium electron distribution function ${ }^{6}$ to be the leading causes of MIRO. Other mechanisms based on different physical pictures were also put forward. ${ }^{7-11}$ Although there exist proposals for the explanation of the ZRS that do not invoke negative resistance (NR) instability, ${ }^{12}$ the commonly accepted explanation (the pattern formation model ${ }^{13}$ ) does invoke the NR instability. This model has also been applied to explain the recently observed zero-differential resistance state (ZDRS) of 2DES in response to a dc excitation above a threshold value at low temperature in a strong magnetic field. ${ }^{14}$

On the experimental side, the outstanding issues include activated temperature dependence with large energy gaps, ${ }^{15}$ immunity of MIRO and ZRS to the polarity of circular polarization of $\mathrm{MW},{ }^{16}$ absolute negative conductivity, ${ }^{15,17-19}$ MW-intensity suppressing of magnetoresistivity $\left(\rho_{x x}\right),{ }^{17,18}$ frequency quenching of MIRO, ${ }^{20}$ Hall-field-induced resistance oscillations (HIRO) in dc-driven 2DES under relatively strong $^{21}$ and weak ${ }^{22} \mathrm{dc}$ bias, suppression of MIRO and ZRS by in-plane magnetic fields, ${ }^{23}$ and multiphoton processes in the ZRS formation. ${ }^{19,24}$ Theoretical understanding of these findings is limited due to the general lack of experience in theoretical investigation of nonequilibrium quantum phenomena.

Among other issues, the polarization immunity of dc resistance poses a particularly challenging test. Experiment by
Smet et al. ${ }^{16}$ established that MIRO are insensitive to the polarization state of the MW radiation. However, transmission data also show that active cyclotron resonance absorption occurs only when the circular polarization matches the magnetic field orientation (denoted as CRA). This observation is inconsistent with the two most prevailing theories, the impurity and/or phonon assisted inter-Landau level (LL) transitions model $^{3-5}$ and the nonequilibrium distribution function scenario. ${ }^{6}$ These theories predict oscillations with correct period and phase, however, with substantially different amplitudes for different polarizations; the MW photoconductivity of the cyclotron resonance inactive (CRI) state is smaller by a factor $\left[\left(\omega-\omega_{c}\right)^{2}+\Gamma^{2}\right] /\left[\left(\omega+\omega_{c}\right)^{2}+\Gamma^{2}\right]$, where $\Gamma^{-1}$ is a phenomenological electron transport lifetime. The factor reflects the huge difference in the (ac) Drude conductivity for opposite circular polarities. We also note that some theoretical models predict polarization independence of the distribution-function dominant contribution to $\rho_{x x}$ only in the regime of intra-LL transitions, ${ }^{25}$ while others assume a strong enough impurity-induced damping that can quench the polarization dependent factor in the (ac) Drude conductivity. ${ }^{26,27}$

\section{THEORY}

Polarization immunity of the dc resistance posts a big challenge to the understanding of the ZRS. To produce the "same" MIRO and ZRS, there must be an additional mechanism that compensates for the discrepancy in the energy absorption rate between the CRA and CRI states. In this paper, we propose that a spontaneous circular motion of the whole electron fluid previously proposed by $\mathrm{Ng}$ and $\mathrm{Dai}^{28}$ could stabilize the system and minimize the polarization dependence of the oscillatory photoconductivity. The spontaneous circular motion arises in the cyclotron resonance (CR) favorable orientation whenever the intensity of the incident MW radiation exceeds a threshold value.

To see how this could happen, we examine the general transport equation for the center of mass $(\mathrm{CM})$ coordinate $\vec{R}(t)$ of the electron liquid where the effect of impurity is included to the second $\operatorname{order}^{28}$ as follows: 


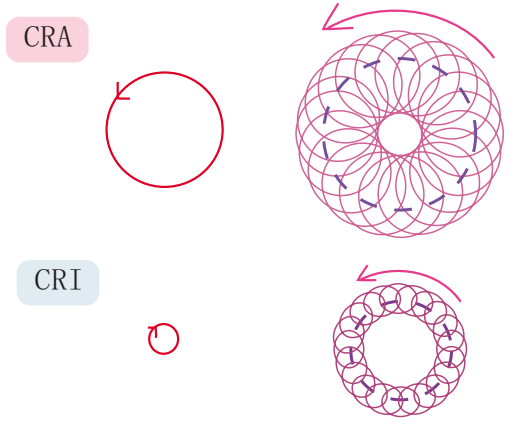

FIG. 1. (Color online) Schematic trajectory of the ac motion of the electron fluid in the absence (left) and presence (right) of the spontaneous circular motion (dashed circle) for CRA (upper) and CRI (lower) states.

$$
\begin{aligned}
m \ddot{\vec{R}}(t)= & (-e)\left(\vec{E}(t)+\frac{1}{c} \dot{\vec{R}}(t) \times \vec{B}\right) \\
& +\alpha \nabla_{\vec{R}(t)} \int_{-\infty}^{t} d t^{\prime} \chi\left[\vec{R}(t)-\vec{R}\left(t^{\prime}\right) ; t-t^{\prime}\right],
\end{aligned}
$$

where $\alpha=n_{i}|u|^{2} / \bar{n}, n_{i}$ is the density of impurity, $|u|^{2}$ measures the strength of the impurity potential, $\bar{n}=N / V$ is the carrier density, and $\chi$ is the (equilibrium) retarded densitydensity response function without the MW term. The equation is derived in the CM frame where the MW field is eliminated and the electron liquid sees moving impurities following paths $\sim-\vec{R}(t)$. After impurity averaging, we obtain Eq. (1). ${ }^{28}$ We note that a similar equation has also been proposed by Lei et al. ${ }^{4}$ The electric field relevant to this phenomenon is of the form $\vec{E}(t)=\vec{E}_{1} \cos (\bar{\omega} t)+\vec{E}_{2} \sin (\bar{\omega} t)+\vec{E}_{d}$, where $\vec{E}_{2}= \pm \hat{z} \times \vec{E}_{1}$ and $\left|\vec{E}_{1}\right|=\left|\vec{E}_{2}\right|=E_{0}$. The first two terms represent a circularly polarized MW radiation with frequency $\bar{\omega}$ and $\vec{E}_{d}$ stands for a small dc bias. The plus or minus sign in the expression of $\vec{E}_{2}$ indicates a CRA or CRI state. For small dc bias $\vec{R}(t)$ can be written as $\vec{R}(t)=\vec{R}_{\mathrm{ac}}(t)+\vec{R}_{\mathrm{dc}}(t)$, where $\vec{R}_{\text {ac }}(t)$ is the (dominant) part induced by the MW field and $\vec{R}_{\mathrm{dc}}(t) \sim \vec{v} t$ is a small dc correction.

Equation (1) suggests that the linear dc resistance is determined completely by $\vec{R}_{\mathrm{ac}}(t)$ since there is nothing else in the equation. As sketched in Fig. 1 (left-hand solid circles), $\vec{R}_{\text {ac }}(t)$ for opposite polarization states have very different amplitudes of motion in the Drude model, and to explain the polarization immunity, this big difference in $\vec{R}_{\mathrm{ac}}(t)$ must be eliminated. A plausible way for this to occur is to have a spontaneous circular motion in the CRA direction for both CRA and CRI states. If this spontaneous circular motion dominates over the Drude motion, the difference in $\vec{R}_{\mathrm{ac}}(t)$ between the CRA and CRI states will be minimized. In reality the composite trajectory of the spontaneous and Drude motion can be rather complicated (Fig. 1, right-hand solid traces) and a self-consistent numerical calculation has to be performed to test this idea.

We study a trial solution of Eq. (1) with

$$
\begin{aligned}
\vec{R}(t)= & \vec{v} \cdot t+\vec{R}_{f}(t)+\vec{R}_{s}(t) \\
= & \vec{v} \cdot t+\vec{A}_{1} \cos (\bar{\omega} t+\delta)+\vec{B}_{1} \sin (\bar{\omega} t+\delta) \\
& +\vec{\alpha}_{1} \cos \left(\omega_{I} t\right)+\vec{\beta}_{1} \sin \left(\omega_{I} t\right)
\end{aligned}
$$

where $\vec{R}_{f}(t)$ describes the CM motion directly coupled to the radiation field and $\vec{R}_{s}(t)$ depicts the spontaneously generated oscillatory mode with frequency $\omega_{I}$. We shall call them "fast" and "slow" modes in the following in view of their frequency difference which will be shown numerically later. $\delta$ is the phase delay of the "fast mode" with respect to the ac driving. Notice that we keep only the fundamental harmonic modes in $\vec{R}_{f}(t)$ and $\vec{R}_{s}(t)$. This can be justified for the fast mode since in the weak radiation limit the size of the circular orbit $R_{c} \sim \sqrt{\vec{A}_{1}^{2}+\vec{B}_{1}^{2}}$ will be much smaller than the magnetic length $l_{c}=\sqrt{c / e B}$ (we set $\hbar=1$ in the following), which is quite long for the $B$ field $(\sim 1 \mathrm{~T})$ relevant to this phenomenon. ${ }^{28}$ Notice that such an argument does not apply to the slow mode whose amplitude has to be determined self-consistently.

We next expand the impurity-scattering-induced damping force $\vec{F}_{I}(t) \equiv \alpha \nabla_{\vec{R}(t)} \int_{-\infty}^{t} d t^{\prime} \chi\left[\vec{R}(t)-\vec{R}\left(t^{\prime}\right) ; t-t^{\prime}\right]$ in a BesselFourier series and keep only the fundamental-frequency oscillating terms. By putting the approximate $\vec{R}(t)$ and $\vec{F}_{I}(t)$ into Eq. (1) and compare, we obtain three force-balance equations

$$
\begin{gathered}
0=-e \vec{E}_{d}-\frac{e}{c} \vec{v} \times \vec{B}+\vec{F}_{v}, \\
m \ddot{\vec{R}}_{f}(t)=-e \vec{E}(t)-\frac{e}{c} \dot{\vec{R}}_{f}(t) \times \vec{B}+\vec{F}_{I}^{(f)}(t), \\
m \ddot{\vec{R}}_{s}(t)=-\frac{e}{c} \dot{\vec{R}}_{s}(t) \times \vec{B}+\vec{F}_{I}^{(s)}(t) .
\end{gathered}
$$

Here

$$
\begin{aligned}
\vec{F}_{v}= & -\alpha \iint d^{d} q \vec{q} \sum_{m, n=-\infty}^{\infty} \operatorname{Im} \chi\left(\vec{q}, \vec{q} \cdot \vec{v}+m \bar{\omega}+n \omega_{I}\right) \\
& \times J_{m}^{2}(z(\vec{q})) J_{n}^{2}(y(\vec{q}))
\end{aligned}
$$

is the time-averaged damping force, where $z(\vec{q})^{2}=\left(\vec{q} \cdot \vec{A}_{1}\right)^{2}$ $+\left(\vec{q} \cdot \vec{B}_{1}\right)^{2}$ and $y(\vec{q})^{2}=\left(\vec{q} \cdot \vec{\alpha}_{1}\right)^{2}+\left(\vec{q} \cdot \vec{\beta}_{1}\right)^{2}$. For the "fast mode," $\vec{F}_{I}^{(f)}(t) \sim \vec{H}_{1} \cos (\bar{\omega} t+\delta)+\vec{G}_{1} \sin (\bar{\omega} t+\delta)$, where $\vec{H}_{1} \equiv \pi^{(1)} \cdot \vec{A}_{1}$ $-\pi^{(2)} \cdot \vec{B}_{1}$ and $\vec{G}_{1} \equiv \pi^{(1)} \cdot \vec{B}_{1}+\pi^{(2)} \cdot \vec{A}_{1}$, whereas for the "slow mode," $\vec{F}_{I}^{(s)}(t) \sim \vec{h}_{1} \cos \left(\omega_{I} t\right)+\vec{g}_{1} \sin \left(\omega_{I} t\right)$, where $\vec{h}_{1}$ $\equiv \pi^{(1)^{\prime}} \cdot \vec{\alpha}_{1}-\pi^{(2)^{\prime}} \cdot \vec{\beta}_{1}$ and $\vec{g}_{1} \equiv \pi^{(1)^{\prime}} \cdot \vec{\beta}_{1}+\pi^{(2)^{\prime}} \cdot \vec{\alpha}_{1}$.

The $\pi^{\prime}$ s are given by

$$
\begin{aligned}
\pi^{(1)}= & \frac{\alpha}{2} \iint d^{d} q q^{2} \sum_{m, n=-\infty}^{\infty} \operatorname{Re} \chi\left(\vec{q}, \vec{q} \cdot \vec{v}+m \bar{\omega}+n \omega_{I}\right) \\
& \times\left[J_{m}(z(\vec{q})) \frac{J_{m}^{\prime}(z(\vec{q}))}{z(\vec{q})}\right] J_{n}^{2}(y(\vec{q})),
\end{aligned}
$$




$$
\begin{aligned}
\pi^{(2)}= & \frac{\alpha}{2} \iint d^{d} q q^{2} \sum_{m, n=-\infty}^{\infty} \operatorname{Im} \chi(\vec{q}, \vec{q} \cdot \vec{v}+m \bar{\omega} \\
& \left.+n \omega_{I}\right) m\left[\frac{J_{m}(z(\vec{q}))}{z(\vec{q})}\right]^{2} J_{n}^{2}(y(\vec{q})),
\end{aligned}
$$

and $\pi^{(1)^{\prime}}, \pi^{(2)^{\prime}}$ can be obtained from $\pi^{(1)}, \pi^{(2)}$ by simply interchanging $z \leftrightarrow y, m \leftrightarrow n$, and $\bar{\omega} \leftrightarrow \omega_{I}$. We shall consider the limit $\vec{v} \rightarrow 0$ when calculating $\pi$ in the following since we are interested in the linear-response dc current.

After some algebra we obtain the equation of motion for fast mode

$$
\sqrt{\left[m \bar{\omega}^{2}\left(1 \mp \omega_{c} / \bar{\omega}\right)+\pi^{(1)}\right]^{2}+\left[\pi^{(2)}\right]^{2}} A_{1}=e E_{0},
$$

where - or + corresponds to a CRA or CRI state. This equation is of the Drude form, describing an oscillation that is driven by an ac electric field and acted on by an impurityinduced friction $\pi^{(2)}$. The friction depends on the amplitudes of both fast and slow modes $[z(\vec{q})$ and $y(\vec{q})]$. A corresponding reactive correction $\pi^{(1)}$ also appears which can be interpreted as a mass correction. ${ }^{28}$ The phase delay $\delta$ is given by $\tan \delta=\pi^{(2)} /\left[m \bar{\omega}^{2}\left(1 \mp \omega_{c} / \bar{\omega}\right)+\pi^{(1)}\right]$. Notice the explicit polarization dependence of $A_{1}$ in Eq. (8).

Since there is no external driving force, the spontaneously generated slow mode is determined by the self-sustainability requirement that the corresponding frictional force vanishes, i.e.,

$$
\pi^{(2)^{\prime}}=0 \text {. }
$$

Putting it into Eq. (5) we obtain another equation,

$$
-m \omega_{I}^{2}\left(1-\omega_{c} / \omega_{I}\right) \alpha_{1}=\pi^{(1)^{\prime}} \alpha_{1} .
$$

These two equations determine self-consistently the amplitude and frequency of the slow mode. Note that the spontaneous circular motion is always in the CR-favorable direction, independent of the polarization of the MW radiation. Polarization dependence enters only indirectly through $z(\vec{q})$ which appears in both $\pi^{(1)^{\prime}}$ and $\pi^{(2)^{\prime}}$.

Equations (8)-(10) form a set of self-consistent equations determining the fast and slow mode amplitudes $A_{1}, \alpha_{1}$ and the slow mode frequency $\omega_{I}$. These equations are solved numerically. The longitudinal dc resistance $R_{x x}=$ $-\lim _{\vec{v} \rightarrow 0}\left[v^{-2} \vec{F}_{v} \cdot \vec{v}\right]$ is computed afterward.

\section{NUMERICAL RESULTS}

We have employed the density-density response function of noninteracting electron gas in a constant magnetic field ${ }^{29}$ in our calculation, with Landau levels (LLs) broadened phenomenologically into Lorentzians, i.e., $\delta\left(\varepsilon-n \omega_{c}\right)$ $\rightarrow \pi^{-1} \Gamma /\left[\left(\varepsilon-n \omega_{c}\right)^{2}+\Gamma^{2}\right]$. Since experimentally $\bar{\omega}$ $\sim 200 \mathrm{GHz}$ is fixed and the $B$ field is swept, we use $\bar{\omega}$ as the basic scale, setting $T \sim \bar{\omega}, \Gamma(T) \sim 0.2 \bar{\omega}, E_{F} \sim 10 \bar{\omega}$. We keep 20 LLs in our calculation, which is consistent with the low field $(B \sim 1 \mathrm{~T})$ and intermediate temperature $(T \sim 1 \mathrm{~K})$ setting in experiment. We shall vary the magnetic field $B$ and use the frequency ratio $\omega_{n} \equiv \bar{\omega} / \omega_{c}$ as the abscissa in present-
TABLE I. The numerical range of the self-consistently determined $d, c$, and $x$ for CRA and CRI states in the regime $\omega_{n} \sim 1-2$.

\begin{tabular}{lcccc}
\hline \hline Polarity & Frequency range & $d$ & $c$ & $x$ \\
\hline$\langle$ CRA $\rangle$ & $\mathrm{NR}\left(\omega_{n}: 1.1-1.5\right)$ & $0.1-0.2$ & $0.2-0.4$ & $\sim 0.3$ \\
& $\operatorname{PR}\left(\omega_{n}: 1.5-1.9\right)$ & $0.2-0.3$ & $0.2-0.4$ & $\sim 1.1$ \\
$\langle\mathrm{CRI}\rangle$ & $\left(\omega_{n}: 1.1-2.0\right)$ & $\sim 0.1$ & $0.03-0.08$ & $\sim 0.3$ \\
\hline \hline
\end{tabular}

ing our results. We also define normalized radiation intensity $I_{N} \equiv\left(e E_{0} / m^{*} \bar{\omega}^{2} \bar{l}\right)^{2}$, where $\bar{l} \equiv \sqrt{\omega_{c} / \bar{\omega}} l_{c} \quad\left(m^{*}=0.068 m_{0}\right.$ is the effective mass of conduction band electrons in GaAs) as well as normalized amplitudes $c \equiv A_{1} / \bar{l}, d \equiv \alpha_{1} / \bar{l}$ and renormalized frequency $x \equiv \omega_{I} / \omega_{c}$ in our calculation.

To further simplify the calculation and analysis, we make another approximation of keeping only $|n|,|m| \leq 1$ terms (zero- and one-photon processes) in the impurity induced forces in our calculation; this is consistent with keeping only fundamental harmonics in our trial trajectory. Correspondingly, we restrict our calculation to the frequency range $\omega_{n}$ $=\bar{\omega} / \omega_{c} \sim 1-2$ since MIRO occur at the weak $B$ field side $\bar{\omega}>\omega_{c}$ and the higher frequency range will be dominated by transitions between higher LLs $(|n|,|m|>1$ processes). The approximation of keeping only single photon processes in the fast mode can be justified in the weak radiation field limit. However, this approximation cannot be justified a priori for the spontaneous (slow) mode. We have estimated the effect of the multiphoton processes associated with the slow mode; in our self-consistent equations, those terms associated with $\operatorname{Im} \chi\left(\vec{q}, \vec{q} \cdot \vec{v}+n \omega_{I}+\bar{\omega}\right)$ are not affected while the terms associated with $\operatorname{Im} \chi\left(\vec{q}, \vec{q} \cdot \vec{v}+n \omega_{I}\right)$ and $\operatorname{Im} \chi\left(\vec{q}, \vec{q} \cdot \vec{v}+n \omega_{I}-\bar{\omega}\right)$ would be enhanced. To mimic these effects we introduce a correction factor $a>1$ that multiplies the latter two terms in $\pi^{(2)^{\prime}}$. The same factor is introduced to the counterparts in $\pi^{(1)^{\prime}}$ and $\vec{F}_{v}\left(R_{x x}\right)$ to ensure consistency in our calculation. As long as $a$ does not deviate too much from $a=1$, the qualitative behavior of the solutions remains unaltered. We shall present our calculation results with $a=1.2$ in the following.

Numerically we find that we may roughly divide the region $\omega_{n} \sim 1-2$ into a negative resistance (NR) region that centers about $\omega_{n} \sim 1.2$, and a positive resistance (PR) region that peaks at $\omega_{n} \sim 1.7$. For radiation intensity $\left(I_{N}\right)_{\text {th }}<I_{N}$ $<\left(I_{N}\right)_{\text {multi- } \bar{\omega}}$, where $\left(I_{N}\right)_{\text {th }} \sim 0.001$ numerically (corresponding to $\left.E_{0} \sim 7 \mathrm{~V} / \mathrm{cm}\right)$ is a threshold value above which the slow mode appears and $\left(I_{N}\right)_{\text {multi- } \bar{\omega}} \sim 0.02\left(E_{0} \sim 32 \mathrm{~V} / \mathrm{cm}\right)$ is a value where multiphoton processes of MW radiation become important, we find that the solutions for $c, d$, and $x$ fall within the ranges listed in Table I. Notice that the amplitude of the fast mode $(c)$ in the CRI state is much smaller than that in the CRA state as expected. However, the amplitudes of the slow mode $(d)$ in the two polarization states are comparable. The low-frequency slow mode $\left(\omega_{I} \sim 0.3 \omega_{c}\right)$ appears in the whole frequency range of the CRI state and the NR region of the CRA state. Unexpectedly, $d \neq 0$ solutions also exist in the PR region of the CRA state with rather large amplitude and near-CR frequency.

The effect of the slow mode on the polarization dependence of dc resistance and the ZRS can be roughly under- 

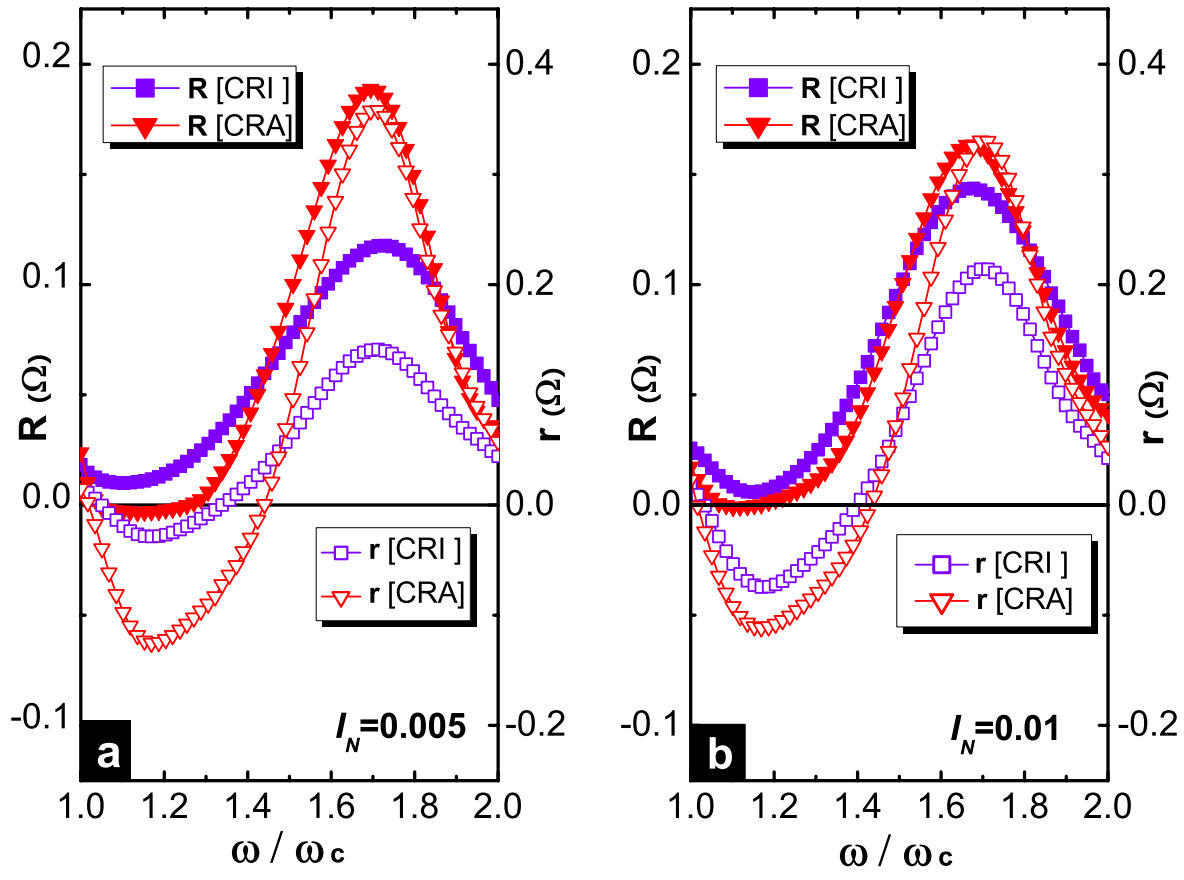

FIG. 2. (Color online) dc resistance versus frequency ratio for CRA and CRI states under two values of radiation intensity: (a) $I_{N}=0.005$ (moderate intensity) and (b) $I_{N}=0.01$ (high intensity). $(R)$ — with slow mode included in calculation; $(r)$-slow mode is excluded. stood as follows: when the incident radiation is sufficiently strong and the NR instability shows up, a spontaneous circular motion is generated in the electron fluid in the CR favorable direction. Mathematically, low-frequency fictitious photons represented by $n \neq 0$ processes are spontaneously generated and additional photon-assisted transport channels open up. As a result normal dissipation is enhanced and NR is cured. This mechanism dominates the whole frequency range of the CRI state and the NR region of the CRA state. The spontaneous circular motion also turns the original fastrotating CRA orbit (left-hand solid circle of Fig. 1) into the "lace" of a slowly rotating $\left(\omega_{I}\right)$ orbit (right-hand dashed circle). The strong mixing of these two orbits destroys the identity of well-defined Landau orbits and suppresses photon-assisted scattering in the CRA state, thus drawing the two states closer to each other. Mathematically, the suppression is reflected in the increase in the argument $(y(\vec{q}))$ of Bessel functions.

We show in Fig. 2 the calculated dc resistance versus frequency ratio both with $(R)$ and without $(r)$ slow mode included in our model. Notice the different plotting scales for the two calculated resistances in the figure. We see that when the radiation is of moderate intensity $I_{N}=0.005\left(E_{0}\right.$ $\sim 16 \mathrm{~V} / \mathrm{cm}$ ) [Fig. 2(a)], the NR region becomes pronounced in the absence of the slow mode for both CRA and CRI states and the disparity in the oscillation amplitude of the two polarization states is large.

The entering of the slow mode suppresses the oscillation amplitudes, especially for the CRA state, which effectively reduces the amplitude discrepancy between the two polarizations; meanwhile the NR instability is almost completely healed by the $\omega_{I}$-photon-excited inter-LL transitions in our simple model. As the radiation strength further increases to $I_{N}=0.01\left(E_{0} \sim 22 \mathrm{~V} / \mathrm{cm}\right) \quad[$ Fig. 2(b)], oscillation becomes stronger for the CRI state but is approaching saturation for the CRA state in the absence of the slow mode. In this case, the suppression effect plus the photon-assisted transport pro- cesses associated with the slow mode bring the oscillatory shape of the two polarization states even closer and lift the $\mathrm{NR}$ region to ZR for both polarities. We note also that at the $B$ field region near $\mathrm{CR}$ where significant absorption takes place, no $d \neq 0$ solution is found in our calculation and the oscillation amplitudes of CRA and CRI curves are considerably different, in agreement with experiment. ${ }^{16}$

\section{CONCLUSION}

We have proposed and demonstrated numerically that a spontaneous slowly rotating circulating current in magnetized 2DES will be generated when the incident circularly polarized MW radiation exceeds certain threshold intensity. The spontaneous mode can cure the NR problem and provides a plausible explanation for the observed ZRS and the polarization immunity of dc resistance. Our numerical calculation shows that the difference in $\vec{R}_{\mathrm{ac}}(t)$ between the CRA and CRI states persists even in the presence of the spontaneous mode (due to the mixing with the Drude motion) and our result is only in qualitative agreement with experiment. Since our calculation is considerably simplified with many approximations employed, it is not clear whether a more complete numerical calculation will reduce further the difference in $\vec{R}_{\text {ac }}(t)$ between the CRA and CRI states or whether some new physical mechanism needs to be included to understand the experimental results quantitatively. Nevertheless, we believe that our theory has provided a promising starting point to investigate the physics behind the ZRS phenomenon and the associated polarization immunity.

\section{ACKNOWLEDGMENT}

We acknowledge support from HKUGC through Grant No. CA05/06.SC04. 
${ }^{1}$ R. G. Mani, J. H. Smet, K. von Klitzing, V. Narayanamurti, W. B. Johnson, and V. Umansky, Nature (London) 420, 646 (2002).

${ }^{2}$ M. A. Zudov, R. R. Du, L. N. Pfeiffer, and K. W. West, Phys. Rev. Lett. 90, 046807 (2003).

${ }^{3}$ A. C. Durst, S. Sachdev, N. Read, and S. M. Girvin, Phys. Rev. Lett. 91, 086803 (2003).

${ }^{4}$ X. L. Lei and S. Y. Liu, Phys. Rev. Lett. 91, 226805 (2003).

${ }^{5}$ M. G. Vavilov and I. L. Aleiner, Phys. Rev. B 69, 035303 (2004)

${ }^{6}$ I. A. Dmitriev, M. G. Vavilov, I. L. Aleiner, A. D. Mirlin, and D. G. Polyakov, Phys. Rev. B 71, 115316 (2005).

${ }^{7}$ Junren Shi and X. C. Xie, Phys. Rev. Lett. 91, 086801 (2003).

${ }^{8}$ P. H. Rivera and P. A. Schulz, Phys. Rev. B 70, 075314 (2004).

${ }^{9}$ S. A. Mikhailov, Phys. Rev. B 70, 165311 (2004).

${ }^{10}$ C. Joas, M. E. Raikh, and F. von Oppen, Phys. Rev. B 70, 235302 (2004).

${ }^{11}$ J. Iñarrea and G. Platero, Phys. Rev. Lett. 94, 016806 (2005).

${ }^{12}$ D. H. Lee and J. M. Leinaas, Phys. Rev. B 69, 115336 (2004).

${ }^{13}$ A. V. Andreev, I. L. Aleiner, and A. J. Millis, Phys. Rev. Lett. 91, 056803 (2003).

${ }^{14}$ A. A. Bykov, J. Q. Zhang, S. Vitkalov, A. K. Kalagin, and A. K. Bakarov, Phys. Rev. Lett. 99, 116801 (2007).

${ }^{15}$ R. L. Willett, L. N. Pfeiffer, and K. W. West, Phys. Rev. Lett. 93, 026804 (2004).

${ }^{16}$ J. H. Smet, B. Gorshunov, C. Jiang, L. Pfeiffer, K. West, V. Umansky, M. Dressel, R. Meisels, F. Kuchar, and K. von Klitzing, Phys. Rev. Lett. 95, 116804 (2005).
${ }^{17}$ S. A. Studenikin, M. Potemski, A. Sachrajda, M. Hilke, L. N. Pfeiffer, and K. W. West, Phys. Rev. B 71, 245313 (2005).

${ }^{18}$ R. G. Mani, V. Narayanamurti, K. von Klitzing, J. H. Smet, W. B. Johnson, and V. Umansky, Phys. Rev. B 69, 161306(R) (2004).

${ }^{19}$ M. A. Zudov, R. R. Du, L. N. Pfeiffer, and K. W. West, Phys. Rev. B 73, 041303(R) (2006).

${ }^{20}$ S. A. Studenikin et al., Phys. Rev. B 76, 165321 (2007).

${ }^{21}$ C. L. Yang, J. Zhang, R. R. Du, J. A. Simmons, and J. L. Reno, Phys. Rev. Lett. 89, 076801 (2002).

${ }^{22}$ W. Zhang, H. S. Chiang, M. A. Zudov, L. N. Pfeiffer, and K. W. West, Phys. Rev. B 75, 041304(R) (2007); J. Q. Zhang, S. Vitkalov, A. A. Bykov, A. K. Kalagin, and A. K. Bakarov, ibid. 75, 081305(R) (2007).

${ }^{23}$ C. L. Yang, R. R. Du, L. N. Pfeiffer, and K. W. West, Phys. Rev. B 74, 045315 (2006).

${ }^{24}$ M. A. Zudov, R. R. Du, L. N. Pfeiffer, and K. W. West, Phys. Rev. Lett. 96, 236804 (2006).

${ }^{25}$ C. Joas, J. Dietel, and Felix von Oppen, Phys. Rev. B 72, 165323 (2005).

${ }^{26}$ J. Iñarrea and G. Platero, Phys. Rev. B 76, 073311 (2007).

${ }^{27}$ I. A. Dmitriev, A. D. Mirlin, and D. G. Polyakov, Phys. Rev. B 75, 245320 (2007).

${ }^{28}$ T. K. Ng and L. Dai, Phys. Rev. B 72, 235333 (2005).

${ }^{29}$ C. S. Ting, S. C. Ying, and J. J. Quinn, Phys. Rev. B 16, 5394 (1977). 